\title{
An extension of a theorem of Marcinkiewicz and Zygmund on differentiability
}

\author{
by
}

S. N. Mukhopadhy ay and S. Mitra (Burdwan)

\begin{abstract}
Let $f$ be a measurable function such that $\Delta_{k}(x, h ; f)=O\left(|h|^{\lambda}\right)$ at each point $x$ of a set $E$, where $k$ is a positive integer, $\lambda>0$ and $\Delta_{k}(x, h ; f)$ is the symmetric difference of $f$ at $x$ of order $k$. Marcinkiewicz and Zygmund [5] proved that if $\lambda=k$ and if $E$ is measurable then the Peano derivative $f_{(k)}$ exists a.e. on $E$. Here we prove that if $\lambda>k-1$ then the Peano derivative $f_{([\lambda])}$ exists a.e. on $E$ and that the result is false if $\lambda=k-1$; it is further proved that if $\lambda$ is any positive integer and if the approximate Peano derivative $f_{(\lambda), \text { a }}$ exists on $E$ then $f_{(\lambda)}$ exists a.e. on $E$.
\end{abstract}

1. Introduction. Let $f$ be a real-valued function defined in some neighbourhood of $x$. Then $f$ is said to have Peano derivative (resp. approximate Peano derivative) at $x$ of order $k$ if there exist real numbers $\alpha_{r}, 1 \leq r \leq k$, depending on $x$ and $f$ only such that

$$
f(x+h)=f(x)+\sum_{r=1}^{k} \frac{h^{r}}{r !} \alpha_{r}+\frac{h^{k}}{k !} \varepsilon_{k}(x, h, f),
$$

where

$$
\lim _{h \rightarrow 0} \varepsilon_{k}(x, h, f)=0 \quad\left(\text { resp. } \operatorname{limap}_{h \rightarrow 0} \varepsilon_{k}(x, h, f)=0\right) .
$$

The number $\alpha_{k}$ is called the Peano derivative (resp. approximate Peano derivative) of $f$ at $x$ of order $k$ and is denoted by $f_{(k)}(x)$ (resp. $f_{(k), a}(x)$ ). For convenience we shall write $\alpha_{0}=f(x)=f_{(0)}(x)=f_{(0), \mathrm{a}}(x)$.

Suppose that $f$ has Peano derivative (resp. approximate Peano derivative) at $x$ of order $k-1$. For $h \neq 0$ we write

$$
\omega_{k}(x, h ; f)=\omega_{k}(x, h)=\frac{k !}{h^{k}}\left[f(x+h)-\sum_{r=0}^{k-1} \frac{h^{r}}{r !} \alpha_{r}\right] .
$$

1991 Mathematics Subject Classification: Primary 26A24.

The work of the second author was supported by a CSIR grant of India. 
The upper and lower Peano derivates (resp. approximate Peano derivates) of $f$ at $x$ of order $k$ are defined by

$$
\begin{array}{ll}
\bar{f}_{(k)}(x)=\limsup _{h \rightarrow 0} \omega_{k}(x, h) & \left(\text { resp. } \bar{f}_{(k), \mathrm{a}}(x)=\limsup _{h \rightarrow 0} \operatorname{ap} \omega_{k}(x, h)\right), \\
\underline{f}_{(k)}(x)=\liminf _{h \rightarrow 0} \omega_{k}(x, h) \quad\left(\operatorname{resp} . \underline{f}_{(k), \mathrm{a}}(x)=\liminf _{h \rightarrow 0} \operatorname{ap} \omega_{k}(x, h)\right) .
\end{array}
$$

The symmetric difference of $f$ at $x$ of order $k$, where $k$ is a positive integer, is defined by

$$
\Delta_{k}(x, h)=\Delta_{k}(x, h ; f)=\sum_{i=0}^{k}(-1)^{k-i}\left(\begin{array}{c}
k \\
i
\end{array}\right) f\left(x+i h-\frac{k}{2} h\right) .
$$

Marcinkiewicz and Zygmund proved in a deep theorem (Theorem 1 of [5]) that if $f$ is measurable and if for a positive integer $k$,

$$
\Delta_{k}(x, h ; f)=O\left(|h|^{k}\right) \quad \text { as } h \rightarrow 0,
$$

for $x$ in a measurable set $E$ then $f_{(k)}$ exists a.e. on $E$. For $k=2$ this is proved in [9, II, p. 78, Theorem 4.30]. For general $k$ the proof is very long and involved (it is worth mentioning that the proof offered by Marcinkiewicz and Zygmund has a lacuna filled by Fejzic and Weil [3]).

The purpose of the present paper is to extend this result. In fact we prove in Theorem 3.1 that if $f$ is measurable and if for a positive integer $k$,

$$
\Delta_{k}(x, h ; f)=O\left(|h|^{\lambda}\right) \quad \text { as } h \rightarrow 0
$$

for $x$ in a set $E$ (not necessarily uniformly), where $\lambda>k-1$, then $f_{([\lambda])}$ exists a.e. on $E,[\lambda]$ denoting the greatest integer not exceeding $\lambda$. For $\lambda=k$ this gives the result of Marcinkiewicz and Zygmund cited above. Also we show in Theorem 3.2 that this result is not true for $\lambda=k-1$. Thirdly, in Theorem 3.4 we show that if we further assume that the approximate Peano derivative $f_{(k-1) \text {, a }}$ exists on $E$ then the above result is true for $\lambda=k-1$. In fact, we prove in Theorem 3.4 that if $f$ is measurable and if

$$
\Delta_{k}(x, h ; f)=O\left(|h|^{p}\right) \quad \text { as } h \rightarrow 0
$$

for every $x$ in a set $E$, where $k$ and $p$ are positive integers, and if $f_{(p), \text { a }}$ exists finitely on $E$ then $f_{(p)}$ exists a.e. on $E$.

We consider the difference

$$
\begin{aligned}
\widetilde{\Delta}_{1}(x, h) & =\widetilde{\Delta}_{1}(x, h ; f)=f(x+h)-f(x), \\
\widetilde{\Delta}_{n}(x, h) & =\widetilde{\Delta}_{n}(x, h ; f) \\
& =\widetilde{\Delta}_{n-1}(x, 2 h ; f)-2^{n-1} \widetilde{\Delta}_{n-1}(x, h ; f), \quad n \geq 2 .
\end{aligned}
$$

It is known [5] that there are constants $a_{j}, 0 \leq j \leq k$, depending on $j$ 
and $k$ only (with $a_{k}=1$ ) such that

$$
\widetilde{\Delta}_{k}(x, h)=\widetilde{\Delta}_{k}(x, h ; f)=a_{0} f(x)+\sum_{j=1}^{k} a_{j} f\left(x+2^{j-1} h\right), \quad k \geq 1,
$$

the coefficients $a_{j}$ satisfying

$$
\sum_{j=0}^{k} a_{j}=0, \quad \sum_{j=1}^{k} 2^{j s} a_{j}=0, \quad s=1, \ldots, k-1 .
$$

Throughout the paper $\mathbb{R}, \mathbb{N}, \mu, \mu^{*}$ will denote the set of reals, the set of positive integers, Lebesgue measure and Lebesgue outer measure respectively.

Theorem MZ1. Let $f: \mathbb{R} \rightarrow \mathbb{R}$ be measurable and let $f_{(k-1)}(x)$ exist for each $x$ in a measurable set $E \subset \mathbb{R}$. If

$$
\omega_{k}(x, h)=O(1) \quad \text { as } h \rightarrow 0 \text { for } x \in E
$$

then $f_{(k)}$ exists a.e. on $E$.

The above theorem was proved by Denjoy [2] for continuous functions. The theorem in its present form is in Lemma 7 of [5] the proof of which is long and involves the theory of Fourier series and analytic functions. Later a real-variable proof was given by Marcinkiewicz [4] (see also [9, II, p. 76, Theorem 4.24]). A simple and completely different proof is given in $[1$, p. 54 , Corollaries 20 and 21]; see also [6].

THEOREM MZ2. If $f_{(k)}(x)$ exists then there is a number $\lambda_{k}$ depending on $k$ only such that

$$
\lambda_{k} \lim _{u \rightarrow 0} \frac{\widetilde{\Delta}_{k}(x, u ; f)}{u^{k}}=f_{(k)}(x) .
$$

TheOrem MZ3. There are constants $C_{0}, C_{1}, \ldots, C_{2^{k-1}-k}$ such that

$$
\widetilde{\Delta}_{k}(x, h)=\sum_{i=0}^{2^{k-1}-k} C_{i} \Delta_{k}\left(x+\frac{1}{2} k h+i h, h\right) .
$$

Theorems MZ2 and MZ3 are also due to Marcinkiewicz and Zygmund. See Art. 9 and Art. 12 respectively of [5] for the proof.

We need the following definition.

Definition. A function $f$ defined in some neighbourhood of a point $x_{0}$ is said to be smooth at $x_{0}$ if

$$
\Delta_{2}\left(x_{0}, h ; f\right)=o(h) \quad \text { as } h \rightarrow 0,
$$

and $f$ is said to be uniformly smooth on a set $E$ if (1.3) holds uniformly on $E$. 


\section{Auxiliary results}

LEMma 2.1. Let 0 be a point of outer density of $E$, let $\alpha, \beta \in \mathbb{R}$ with $\beta \neq 0$ and let $\varepsilon>0$. For each $u>0$ set

$$
B_{u}=\{v \in[u, 2 u]: \alpha u+\beta v \in E\} .
$$

Then there is a $\delta>0$ such that if $0<u<\delta$ then $\mu^{*}\left(B_{u}\right)>u(1-\varepsilon)$.

This is Lemma 1 of [3].

Theorem 2.2. Let $f: \mathbb{R} \rightarrow \mathbb{R}$ be measurable and let $f_{(k-1)}$ exist on a set $E, k \in \mathbb{N}$. If

$$
\omega_{k}(x, h)=O(1) \quad \text { as } h \rightarrow 0 \text { for } x \in E,
$$

then $f_{(k)}$ exists finitely a.e. on E.

Proof. Let $G$ be the set of all $x$ such that $f_{(k-1)}$ exists. Then $G$ is measurable and $\bar{f}_{(k)}$ and $\underline{f}_{(k)}$ are measurable on $G$ (see [6]). Hence the set

$$
H=\left\{x \in G:-\infty<\underline{f}_{(k)}(x) \leq \bar{f}_{(k)}(x)<\infty\right\}
$$

is measurable. So by Theorem MZ1, $f_{(k)}$ exists finitely a.e. on $H$. Since $E \subset H$, the result follows.

Lemma 2.3. Let $k \in \mathbb{N}$ and let $f: \mathbb{R} \rightarrow \mathbb{R}$ be measurable. Let

$$
\widetilde{\Delta}_{k}(x, u ; f)=O(1) \quad \text { as } u \rightarrow 0
$$

for each $x$ in a set $E \subset \mathbb{R}$. Then $f$ is bounded in some neighbourhood of almost every point of $E$.

Proof. The proof is given in [3, Theorem 2]. We give a proof for completeness.

For each $m \in \mathbb{N}$ let

$$
\begin{aligned}
& E_{m}=\left\{x \in E:\left|\widetilde{\Delta}_{k}(x, u)\right|<m \text { for } 0<|u|<1 / m\right\}, \\
& F_{m}=\{x \in E:|f(x)|<m\} .
\end{aligned}
$$

Since $E=\bigcup_{m}\left(E_{m} \cap F_{m}\right)$, it suffices to prove that $f$ is bounded on some neighbourhood of every point of outer density of $E_{m} \cap F_{m}$. Let $x_{0}$ be such a point; suppose $x_{0}=0$. By Lemma 2.1 there is $\delta$ with $0<\delta<1 / m$ such that if $0<u<\delta$ then

$$
\mu^{*}(B)>u(1-1 /(4 k)) \text { and } \mu^{*}\left(C_{r}\right)>u(1-1 /(4 k)),
$$

where

$$
\begin{aligned}
B & =[u, 2 u] \cap E_{m} \cap F_{m}, \\
C_{r} & =\left\{v \in[u, 2 u]: v+(u-v) / 2^{k-r-1} \in F_{m}\right\}, \quad 0 \leq r \leq k-2 .
\end{aligned}
$$

Fix $0<u<\delta$. Let

$$
D_{r}=\left\{v \in[u, 2 u]:\left|f\left(v+(u-v) / 2^{k-r-1}\right)\right|<m\right\} .
$$


Then $D_{r}$ is measurable and $C_{r} \subset D_{r}$ for $0 \leq r \leq k-2$. Now by the measurability of $D_{r}$,

$$
\mu^{*}\left(B \cap D_{r}\right) \geq(1-2 /(4 k)) u \quad \text { for } 0 \leq r \leq k-2,
$$

and hence applying this argument repeatedly,

$$
\mu^{*}\left(B \cap \bigcap_{r} D_{r}\right) \geq(1-k /(4 k)) u>0 .
$$

Choose $v \in B \cap \bigcap_{r} D_{r}$. Since $v \in E_{m}$ and $\left|(u-v) / 2^{k-1}\right|<u<\delta<1 / m$,

$$
\begin{gathered}
\left|\widetilde{\Delta}_{k}\left(v,(u-v) / 2^{k-1}\right)\right|<m, \\
|f(v)|<m, \quad\left|f\left(v+(u-v) / 2^{k-r-1}\right)\right|<m \quad \text { for } 0 \leq r \leq k-2 .
\end{gathered}
$$

Hence from (1.2),

$$
\begin{aligned}
|f(u)| \leq & \left|\widetilde{\Delta}_{k}\left(v,(u-v) / 2^{k-1}\right)\right|+\left|a_{0} f(v)\right| \\
& +\sum_{j=1}^{k-1}\left|a_{j} f\left(v+2^{j-1}(u-v) / 2^{k-1}\right)\right| \\
\leq & m\left[1+\sum_{j=0}^{k-1}\left|a_{j}\right|\right] .
\end{aligned}
$$

This completes the proof.

Lemma 2.4. Let $k \in \mathbb{N}, \lambda \in \mathbb{R}$ and $\lambda>k-1$. Let $f: \mathbb{R} \rightarrow \mathbb{R}$ be measurable. Let $m \in \mathbb{N}$ and let

$$
E=E_{m}=\left\{x:\left|\Delta_{k}(x, h)\right|<m|h|^{\lambda} \text { for } 0<|h|<1 / m\right\} .
$$

Then

If $k \geq 2$ then

$$
\widetilde{\Delta}_{k}(x, h)=O\left(|h|^{\lambda}\right) \quad \text { as } h \rightarrow 0 \quad \text { a.e. on } E_{m} .
$$

$$
\widetilde{\Delta}_{i}(x, h)=O\left(h^{i}\right) \quad \text { as } h \rightarrow 0 \quad \text { a.e. on } E_{m}, 1 \leq i \leq k-1 .
$$

Proof. Let $x_{0} \in E_{m}$ be a point of outer density of $E_{m}$. We may suppose that $x_{0}=0$. Let $0<\varepsilon<1 / 4^{k}$. Then by Lemma 2.1 there is $\delta$ with $0<\delta<1$ such that if $0<u<\delta$ then

$$
\mu^{*}\left(B_{i j}\right)>(1-\varepsilon) u \quad \text { and } \quad \mu^{*}\left(C_{l}\right)>(1-\varepsilon) u,
$$

where

$$
\begin{aligned}
& B_{i j}=\{v \in[u, 2 u]:(k / 2+j)(u+i(v-u) / k) \in E\}, \\
& 1 \leq i \leq k, 0 \leq j \leq 2^{k-1}-k, \\
& C_{l}=\left\{v \in[u, 2 u]: 2^{l}(u+v) / 2 \in E\right\}, \quad 0 \leq l \leq k-1 .
\end{aligned}
$$


Fix $u \in\left(0, \min \left[\delta /(2 m), 1 /\left(m \cdot 2^{k}\right)\right]\right)$. Set

$$
\begin{gathered}
S_{i j}=\{v \in[u, 2 u]: \\
\left.\quad\left|\Delta_{k}((k / 2+j)(u+i(v-u) / k), u+i(v-u) / k)\right|<m(2 u)^{\lambda}\right\}, \\
T_{l}=\left\{v \in[u, 2 u]:\left|\Delta_{k}\left(2^{l}(u+v) / 2,2^{l}(v-u) / k\right)\right|<m\left(2^{k} u\right)^{\lambda}\right\} .
\end{gathered}
$$

Since $f$ is measurable, the sets $S_{i j}, T_{l}$ are all measurable. Also $B_{i j} \subset S_{i j}$ and $C_{l} \subset T_{l}$. Therefore from (2.1),

$$
\mu\left(S_{i j}\right)>(1-\varepsilon) u \quad \text { and } \quad \mu\left(T_{l}\right)>(1-\varepsilon) u .
$$

Since the complement of $\bigcap_{i} \bigcap_{j} \bigcap_{l}\left(S_{i j} \cap T_{l}\right)$ with respect to $[u, 2 u]$ has measure $\leq 4^{k} \varepsilon u$, we have

$$
\mu\left(\bigcap_{i} \bigcap_{j}\left(S_{i j} \cap T_{l}\right)\right) \geq\left(1-4^{k} \varepsilon\right) u>0 .
$$

Let $v \in \bigcap_{i} \bigcap_{j} \bigcap_{l}\left(S_{i j} \cap T_{l}\right)$. Then since $v \in T_{l}$,

$$
\left|\Delta_{k}\left(2^{l}(u+v) / 2,2^{l}(v-u) / k\right)\right|<m\left(2^{k} u\right)^{\lambda}, \quad 0 \leq l \leq k-1,
$$

and so

$$
\left|\sum_{i=0}^{k}(-1)^{k-i}\left(\begin{array}{c}
k \\
i
\end{array}\right) f\left(2^{l} u+2^{l} i(v-u) / k\right)\right|<m\left(2^{k} u\right)^{\lambda} .
$$

Multiplying by $\left|a_{l+1}\right|$ and adding over $l=0,1, \ldots, k-1$ we have

$$
\left|\sum_{i=0}^{k}(-1)^{k-i}\left(\begin{array}{c}
k \\
i
\end{array}\right) \sum_{l=0}^{k-1} a_{l+1} f\left(2^{l} u+2^{l} i(v-u) / k\right)\right|<m_{1} u^{\lambda},
$$

where

$$
m_{1}=m \sum_{l=0}^{k-1}\left|a_{l+1}\right| \cdot 2^{k \lambda}
$$

and so by (1.2),

$$
\left|\sum_{i=0}^{k}(-1)^{k-i}\left(\begin{array}{l}
k \\
i
\end{array}\right) \widetilde{\Delta}_{k}(0, u+i(v-u) / k)\right|<m_{1} u^{\lambda} .
$$

Also since $v \in S_{i j}$ for all $1 \leq i \leq k$ and $0 \leq j \leq 2^{k-1}-k$,

$$
\begin{aligned}
& \left|\Delta_{k}((k / 2+j)(u+i(v-u) / k), u+i(v-u) / k)\right|<m(2 u)^{\lambda} \\
& \qquad \text { for } 1 \leq i \leq k, 0 \leq j \leq 2^{k-1}-k .
\end{aligned}
$$

Hence from Theorem MZ3, 


$$
\begin{aligned}
& \left|\widetilde{\Delta}_{k}(0, u+i(v-u) / k)\right| \\
& \quad \leq \sum_{j=0}^{2^{k-1}-k}\left|C_{j}\right| \cdot\left|\Delta_{k}((k / 2+j)(u+i(v-u) / k), u+i(v-u) / k)\right| \\
& \quad \leq m_{2} u^{\lambda} \quad \text { for } 1 \leq i \leq k,
\end{aligned}
$$

where

$$
m_{2}=\sum_{j=0}^{2^{k-1}-k}\left|C_{j}\right| \cdot 2^{\lambda}
$$

From (2.2) and (2.3),

$$
\left|\widetilde{\Delta}_{k}(0, u)\right|<M u^{\lambda}
$$

where

$$
M=m_{1}+m_{2} \sum_{i=1}^{k}\left(\begin{array}{l}
k \\
i
\end{array}\right) .
$$

Thus the lemma is proved when $u>0$. The proof is similar when $u$ is negative. This completes the proof of the first part.

By the first part and by Lemma 2.3, $f$ is bounded in some neighbourhood of almost all points of $E$. Let $S$ be the set of all points $x \in E$ such that $f$ is bounded in some neighbourhood of $x$ and

$$
\widetilde{\Delta}_{k}(x, h)=O\left(|h|^{\lambda}\right) \quad \text { as } h \rightarrow 0 .
$$

Then $\mu^{*}(S)=\mu^{*}(E)$. We shall show that for each $x \in S$,

$$
\widetilde{\Delta}_{i}(x, h)=O\left(h^{i}\right) \quad \text { as } h \rightarrow 0, i=1, \ldots, k-1,
$$

and this will complete the proof.

Let $x \in S$. We may suppose that $x=0$. Then by (2.4) there are $M>$ 0 and $\delta>0$ such that $f$ is bounded in $[-\delta, \delta]$ and if $0<|u| \leq \delta$ then using (1.1),

$$
\left|\widetilde{\Delta}_{k-1}(0, u)-2^{k-1} \widetilde{\Delta}_{k-1}(0, u / 2)\right|<M|u|^{\lambda} .
$$

Replacing $u$ successively by $u / 2, u / 2^{2}, \ldots, u / 2^{n-1}$, we have

$$
\begin{aligned}
& \left|\widetilde{\Delta}_{k-1}(0, u / 2)-2^{k-1} \widetilde{\Delta}_{k-1}\left(0, u / 2^{2}\right)\right|<M|u / 2|^{\lambda}, \\
& \vdots \\
& \left|\widetilde{\Delta}_{k-1}\left(0, u / 2^{n-1}\right)-2^{k-1} \widetilde{\Delta}_{k-1}\left(0, u / 2^{n}\right)\right|<M\left|u / 2^{n-1}\right|^{\lambda} .
\end{aligned}
$$

Multiplying these inequalities by $1,2^{k-1}, 2^{2(k-1)}, \ldots, 2^{(n-1)(k-1)}$ respectively and adding we get

$$
\left|\widetilde{\Delta}_{k-1}(0, u)-2^{n(k-1)} \widetilde{\Delta}_{k-1}\left(0, u / 2^{n}\right)\right|<M|u|^{\lambda} \sum_{i=0}^{n-1}\left(1 / 2^{\lambda-k+1}\right)^{i} .
$$


Hence

$$
\begin{aligned}
& \left|2^{n(k-1)} \widetilde{\Delta}_{k-1}\left(0, u / 2^{n}\right) / u^{k-1}\right| \\
& \leq M|u|^{\lambda-k+1} \sum_{i=0}^{n-1}\left(1 / 2^{\lambda-k+1}\right)^{i}+\left|\widetilde{\Delta}_{k-1}(0, u) / u^{k-1}\right| \quad \text { if } 0<|u| \leq \delta .
\end{aligned}
$$

So by (1.2) and (2.6) there is a constant $M_{2}$ such that

$$
\left|2^{n(k-1)} \widetilde{\Delta}_{k-1}\left(0, u / 2^{n}\right) / u^{k-1}\right| \leq M_{2} \quad \text { for } \delta / 2^{k} \leq|u| \leq \delta / 2^{k-1} .
$$

Now for each $\omega$ satisfying $0<|\omega| \leq \delta / 2^{k}$ there is a positive integer $n$ such that $2^{n}|\omega| \in\left[\delta / 2^{k}, \delta / 2^{k-1}\right]$ and hence putting $2^{n} \omega=u$ we get, from (2.7),

$$
\left|\widetilde{\Delta}_{k-1}(0, \omega) / \omega^{k-1}\right| \leq M_{2}
$$

Thus

$$
\widetilde{\Delta}_{k-1}(0, u)=O\left(u^{k-1}\right),
$$

which proves (2.5) for $i=k-1$. We suppose that

$$
\widetilde{\Delta}_{j}(0, u)=O\left(u^{j}\right) \quad \text { for } 1<j \leq k-1 .
$$

Then there is $L>0$ such that for small $|u|$ we have as above

$$
\begin{aligned}
& \left|\widetilde{\Delta}_{j-1}(0, u)-2^{j-1} \Delta_{j-1}(0, u / 2)\right|<L|u|^{j}, \\
& \left|\widetilde{\Delta}_{j-1}(0, u / 2)-2^{j-1} \widetilde{\Delta}_{j-1}\left(0, u / 2^{2}\right)\right|<L|u / 2|^{j}, \\
& \vdots \\
& \left|\widetilde{\Delta}_{j-1}\left(0, u / 2^{n-1}\right)-2^{j-1} \widetilde{\Delta}_{j-1}\left(0, u / 2^{n}\right)\right|<L\left|u / 2^{n-1}\right|^{j} .
\end{aligned}
$$

Multiplying these inequalities by $1,2^{j-1}, 2^{2(j-1)}, \ldots, 2^{(n-1)(j-1)}$ respectively and adding we get

$$
\left|\widetilde{\Delta}_{j-1}(0, u)-2^{n(j-1)} \widetilde{\Delta}_{j-1}\left(0, u / 2^{n}\right)\right|<2 L|u|^{j} .
$$

Hence

$$
\left|2^{n(j-1)} \widetilde{\Delta}_{j-1}\left(0, u / 2^{n}\right) / u^{j-1}\right|<2 L|u|+\left|\widetilde{\Delta}_{j-1}(0, u) / u^{j-1}\right| .
$$

Now just as (2.8) is deduced from (2.6) the following can be deduced from (2.10):

$$
\widetilde{\Delta}_{j-1}(0, u)=O\left(u^{j-1}\right) .
$$

Thus if (2.9) holds then (2.11) holds. Since (2.8) holds the proof is complete by induction.

Lemma 2.5. Under the hypothesis of Lemma $2.4, f_{([\lambda])}$ exists and is finite a.e. on $E,[\lambda]$ denoting the greatest integer not exceeding $\lambda$. 
Proof. First we consider the case $[\lambda]=k-1$. If $k=1$ then $[\lambda]=0$ and so the result is trivially true. Suppose $k \geq 2$. Then by Lemma 2.4 ,

$$
\widetilde{\Delta}_{i}(x, u)=O\left(u^{i}\right) \quad \text { as } u \rightarrow 0 \text { for } 1 \leq i \leq k-1
$$

at almost all points of $E$. So taking $i=1$, by the Denjoy-Young-Saks Theorem [7, p. 271], $f^{\prime}$ exists and is finite a.e. on $E$. If $k=2$ then $[\lambda]=1$ and so the result follows. Therefore we suppose $k \geq 3$. Then as above $f^{\prime}$ exists and is finite a.e. on $E$. Suppose that $f_{(r)}(x)$ exists and is finite a.e. on $E$ for a fixed $r, 1 \leq r<k-1$. Let $S \subset E$ be the set of points $x$ such that $f_{(r)}(x)$ exists and (2.12) holds. Then $\mu^{*}(S)=\mu^{*}(E)$. Let $x \in S$ be fixed. We may suppose that

$$
f_{(i)}(x)=0 \quad \text { for } i=0,1, \ldots, r .
$$

Then from Theorem MZ2,

$$
\lim _{u \rightarrow 0} \widetilde{\Delta}_{i}(x, u) / u^{i}=0 \quad \text { for } i=1, \ldots, r .
$$

Since $\widetilde{\Delta}_{r+1}(x, u)=O\left(u^{r+1}\right)$, there are $M>0$ and $\delta>0$ such that

$$
\left|\widetilde{\Delta}_{r}(x, 2 u)-2^{r} \widetilde{\Delta}_{r}(x, u)\right|<M|u|^{r+1} \quad \text { for } 0<|u|<\delta .
$$

Replacing $u$ by $u / 2, u / 2^{2}, \ldots, u / 2^{n}$ successively and then multiplying the terms so obtained by $2^{r}, 2^{2 r}, \ldots, 2^{n r}$ respectively and then adding them with (2.14) we get, as in Lemma 2.4,

$$
\left|\widetilde{\Delta}_{r}(x, 2 u)-2^{r(n+1)} \widetilde{\Delta}_{r}\left(x, u / 2^{n}\right)\right|<2 M|u|^{r+1} .
$$

Dividing by $|u|^{r}$ and letting $n \rightarrow \infty$ gives, by (2.13),

$$
\left|\widetilde{\Delta}_{r}(x, 2 u)\right| \leq 2 M|u|^{r+1} \quad \text { for }|u|<\delta,
$$

that is, $\widetilde{\Delta}_{r}(x, u)=O\left(u^{r+1}\right)$ as $u \rightarrow 0$. Repeating these arguments we ultimately get $\widetilde{\Delta}_{1}(x, u)=O\left(u^{r+1}\right)$ as $u \rightarrow 0$, that is,

$$
f(x+u)=O\left(u^{r+1}\right) \quad \text { as } u \rightarrow 0 .
$$

Since $x \in S$ is arbitrary, by Theorem $2.2, f_{(r+1)}$ exists a.e. on $S$, that is, a.e. on $E$. So by induction $f_{(k-1)}$ exists finitely a.e. on $E$. Thus the result is true in this case.

To complete the proof we suppose that the result is true for $[\lambda]=k-$ $1+r, r \geq 0$. Let $[\lambda]=k+r$. Then $\lambda=k+r+\alpha$, where $0 \leq \alpha<1$. Since

$$
\left|\Delta_{k}(x, u)\right|<m|u|^{\lambda} \quad \text { for } 0<|u|<1 / m, x \in E,
$$

we have

$$
\left|\Delta_{k}(x, u)\right|<m|u|^{k-1+r+\alpha} \quad \text { for } 0<|u|<1 / m, x \in E .
$$

Therefore, since the result is true for $[\lambda]=k-1+r$, we conclude that $f_{(k-1+r)}$ exists and is finite a.e. on $E$. Since $\left|\Delta_{k}(x, u)\right|<m|u|^{\lambda}$ for $0<|u|<1 / m$ 
and $x \in E$ and since $[\lambda]=k+r$,

$$
\left|\Delta_{k}(x, u)\right|<m|u|^{k+r} \quad \text { for } 0<|u|<1 / m, x \in E .
$$

Therefore proceeding as in Lemma 2.4 we conclude that

$$
\widetilde{\Delta}_{k}(x, u)=O\left(u^{k+r}\right) \quad \text { as } u \rightarrow 0
$$

at almost all points of $E$. Let $S$ be the set of points $x$ of $E$ such that $f_{(k-1+r)}(x)$ exists and $(2.16)$ holds. Then $\mu^{*}(S)=\mu^{*}(E)$. Let $x \in S$; we may suppose that $f_{(i)}(x)=0$ for $i=0,1, \ldots, k-1$. Then from Theorem MZ2,

$$
\lim _{u \rightarrow 0} \widetilde{\Delta}_{i}(x, u) / u^{i}=0 \quad \text { for } i=1, \ldots, k-1 .
$$

By (2.16) there are $M>0$ and $\delta>0$ such that

$$
\left|\widetilde{\Delta}_{k-1}(x, 2 u)-2^{k-1} \widetilde{\Delta}_{k-1}(x, u)\right|<M|u|^{k+r} \quad \text { for } 0<|u|<\delta .
$$

Replacing $u$ by $u / 2, u / 2^{2}, \ldots, u / 2^{n}$ successively and then multiplying the inequalities so obtained by $2^{k-1}, 2^{2(k-1)}, \ldots, 2^{n(k-1)}$ respectively and then adding them with $(2.18)$ we get

$$
\left|\widetilde{\Delta}_{k-1}(x, 2 u)-2^{(n+1)(k-1)} \widetilde{\Delta}_{k-1}\left(x, u / 2^{n}\right)\right|<2 M|u|^{k+r} .
$$

Dividing by $|u|^{k-1}$ and letting $n \rightarrow \infty$ we get from this, and from (2.17),

$$
\left|\widetilde{\Delta}_{k-1}(x, 2 u)\right| \leq 2 M|u|^{k+r},
$$

that is, $\widetilde{\Delta}_{k-1}(x, u)=O\left(u^{k+r}\right)$. Repeating these arguments we get $\widetilde{\Delta}_{1}(x, u)$ $=O\left(u^{k+r}\right)$, that is, $f(x+u)=O\left(u^{k+r}\right)$. Since $x \in S$ is arbitrary, by Theorem 2.2, $f_{(k+r)}$ exists a.e. on $S$, that is, a.e. on $E$. This shows that the result is true for $[\lambda]=k+\mathrm{r}$. This completes the proof of the lemma by induction.

\section{Main results}

Theorem 3.1. Let $k \in \mathbb{N}$ and $\lambda \in \mathbb{R}$ be such that $\lambda>k-1$. Let $f: \mathbb{R} \rightarrow \mathbb{R}$ be measurable. If

$$
\Delta_{k}(x, h ; f)=O\left(|h|^{\lambda}\right) \quad \text { as } h \rightarrow 0
$$

for each point $x$ in a set $E \subset \mathbb{R}$ then $f_{([\lambda])}$ exists and is finite a.e. on $E$.

Proof. For each positive integer $m$ let

$$
E_{m}=\left\{x:\left|\Delta_{k}(x, u)\right|<m|u|^{\lambda} \text { for } 0<|u|<1 / m\right\} .
$$

Then $\left\{E_{m}\right\}$ is a non-decreasing sequence and by (3.1), $E \subset \bigcup_{m=1}^{\infty} E_{m}$. By Lemma $2.5, f_{([\lambda])}$ exists and is finite a.e. on $E_{m}$ for each $m$. This completes the proof.

The following theorem shows that Theorem 3.1 is not true for $\lambda=k-1$, $k \geq 2$. 
TheOREM 3.2. For each integer $k \geq 2$ there exists a function $F$ such that

$$
\Delta_{k}(x, h ; F)=o\left(h^{k-1}\right)
$$

uniformly for all $x, F^{(k-2)}$ exists and is continuous for all $x$ but $F_{(k-1)}$ can exist at most on a set of measure zero.

To prove the theorem we need the following lemma.

LEMMA 3.3. Let $k \geq 2$ be an integer, $f$ be locally integrable and uniformly smooth for all $x$ and $F$ be the $(k-2)$ th integral of $f$. Then

$$
\Delta_{k}(x, 2 h ; F)=o\left(h^{k-1}\right)
$$

uniformly for all $x$.

Proof. The case of $k=2$ is trivial. We assume that $k>2$ and $k$ is even. The case of $k$ odd is similar. Let $k=2 \mathrm{~m}$. Since $f$ is uniformly smooth for all $x$, for every $\varepsilon>0$ there exists $\delta>0$, independent of $x$, such that

$$
|(f(x+h)+f(x-h)-2 f(x)) / h|<\varepsilon \quad \text { for } 0<h<\delta \text { and for all } x .
$$

So

$$
-\varepsilon t<f(x+t)+f(x-t)-2 f(x)<\varepsilon t \quad \text { for } 0<t<h<\delta .
$$

Integrating the inequality (3.2) repeatedly $2 m-2$ times over $[0, h]$ we get

$$
\begin{aligned}
-\varepsilon h^{2 m-1} & /(2 m-1) ! \\
& <F(x+h)+F(x-h)-2 \sum_{i=0}^{m-2} \frac{h^{2 i}}{(2 i) !} F^{(2 i)}(x)-2 \frac{h^{2 m-2}}{(2 m-2) !} f(x) \\
& <\varepsilon h^{2 m-1} /(2 m-1) ! .
\end{aligned}
$$

Hence

$$
\begin{array}{r}
{[F(x+h)+F(x-h)] / 2-\sum_{i=0}^{m-2} \frac{h^{2 i}}{(2 i) !} F^{(2 i)}(x)-\frac{h^{2 m-2}}{(2 m-2) !} f(x)} \\
=o\left(h^{2 m-1}\right),
\end{array}
$$

uniformly for all $x$. Now using the relations

$$
\sum_{i=0}^{p}(-1)^{p-i}\left(\begin{array}{c}
p \\
i
\end{array}\right) i^{q}= \begin{cases}0 & \text { if } q=0,1, \ldots, p-1, \\
p ! & \text { if } q=p,\end{cases}
$$

from (3.3) we get 


$$
\begin{aligned}
& \Delta_{2 m}(x, 2 h ; F) \\
&=\sum_{j=0}^{2 m}(-1)^{2 m-j}\left(\begin{array}{c}
2 m \\
j
\end{array}\right) F(x+2 j h-2 m h) \\
&=\sum_{j=0}^{2 m}(-1)^{j}\left(\begin{array}{c}
2 m \\
j
\end{array}\right) F(x-2 j h+2 m h) \\
&=\sum_{j=0}^{2 m}(-1)^{j}\left(\begin{array}{c}
2 m \\
j
\end{array}\right) \frac{1}{2}[F(x+2(m-j) h)+F(x-2(m-j) h)] \\
&=\sum_{j=0}^{2 m}(-1)^{j}\left(\begin{array}{c}
2 m \\
j
\end{array}\right)\left[\sum_{i=0}^{m-2} \frac{[2(m-j) h]^{2 i}}{(2 i) !} F^{(2 i)}(x)+\frac{[2(m-j) h]^{2 m-2}}{(2 m-2) !} f(x)\right] \\
&+o\left(h^{2 m-1}\right) \\
&=\sum_{i=0}^{m-2} \frac{h^{2 i}}{(2 i) !} F^{(2 i)}(x)\left[\sum_{j=0}^{2 m}(-1)^{j}\left(\begin{array}{c}
2 m \\
j
\end{array}\right)(2 m-2 j)^{2 i}\right] \\
&+\left[\frac{(2 h)^{2 m-2}}{(2 m-2) !} f(x) \sum_{j=0}^{2 m}(-1)^{j}\left(\begin{array}{c}
2 m \\
j
\end{array}\right)(m-j)^{2 m-2}\right]+o\left(h^{2 m-1}\right) \\
&= o\left(h^{2 m-1}\right)
\end{aligned}
$$

uniformly for all $x$. This completes the proof.

Proof of Theorem 3.2. Let

$$
f(x)=\sum_{n=1}^{\infty} n^{-1 / 2} b^{-n} \cos \left(b^{n} x\right), \quad b>1 \text { an integer. }
$$

Then $f$ is continuous and uniformly smooth [9, I, p. 47, Theorem 4.10]. For $k=2$, let $F=f$ and for $k>2$ let $F$ be the $(k-2)$ th integral of $f$. We first show that

$$
\lim _{h \rightarrow 0} \Delta_{k-1}(x, h ; F) / h^{k-1}
$$

can exist finitely at most on a set of measure zero. Let $k=2$. Then

$$
\begin{aligned}
\Delta_{1}(x, 2 h ; f) /(2 h) & =[f(x+h)-f(x-h)] /(2 h) \\
& =-\sum_{n=1}^{\infty} n^{-1 / 2} \sin \left(b^{n} x\right)\left[\sin \left(b^{n} h\right) /\left(b^{n} h\right)\right] .
\end{aligned}
$$

If

$$
\lim _{h \rightarrow 0} \Delta_{1}(x, 2 h ; f) /(2 h)
$$


exists finitely on a set of positive measure then from (3.6) the series

$$
-\sum_{n=1}^{\infty} n^{-1 / 2} \sin \left(b^{n} x\right)
$$

is Lebesgue summable on a set of positive measure. Since (3.8) is a lacunary series, by [9, I, p. 203, Theorem 6.4], $\sum_{n=1}^{\infty} 1 / n$ is convergent, which is a contradiction. So (3.7) exists finitely at most on a set of measure zero.

Next suppose $k>2$. We prove that (3.5) can exist finitely at most on a set of measure zero. We suppose that $k$ is even. Let $k=2 m$. Now

$$
\frac{\Delta_{2 m-1}(x, 2 h ; F)}{(2 h)^{2 m-1}}=-\sum_{n=1}^{\infty} n^{-1 / 2} \sin \left(b^{n} x\right)\left(\sin \left(b^{n} h\right) /\left(b^{n} h\right)\right)^{2 m-1} .
$$

If the limit of the left hand side of (3.9) exists on a set of positive measure as $h \rightarrow 0$ then the series $(3.8)$ is $(R, 2 m-1)$ summable and so as in the case of $k=2, \sum_{n=1}^{\infty} 1 / n$ would be convergent, which is a contradiction. Thus the limit of the left hand side of (3.9) as $h \rightarrow 0$ can exist at most on a set of measure zero. If $k$ is odd then it can be similarly proved that (3.5) can exist finitely at most on a set of measure zero.

Now from Lemma 3.3 and the construction of the function $F$ we see that

$$
\Delta_{k}(x, h ; F)=o\left(h^{k-1}\right)
$$

uniformly for all $x$. Also it is clear that $F^{(k-2)}$ exists and is continuous for all $x$. To complete the proof we show that $F_{(k-1)}$ can exist at most on a set of measure zero.

Let, if possible, $F_{(k-1)}$ exist finitely on a set $E$ of positive measure. Then for $x \in E$,

$$
F(x+h)=\sum_{j=0}^{k-1} \frac{h^{j}}{j !} F_{(j)}(x)+o\left(h^{k-1}\right)
$$

and so for $x \in E$, by (3.4),

$$
\begin{aligned}
& \Delta_{k-1}(x, 2 h ; F) \\
& =\sum_{i=0}^{k-1}(-1)^{k-1-i}\left(\begin{array}{c}
k-1 \\
i
\end{array}\right) F(x+2 i h-(k-1) h) \\
& =\sum_{i=0}^{k-1}(-1)^{k-1-i}\left(\begin{array}{c}
k-1 \\
i
\end{array}\right)\left[\sum_{j=0}^{k-1} \frac{(2 i-k+1)^{j} h^{j}}{j !} F_{(j)}(x)+o\left(h^{k-1}\right)\right]
\end{aligned}
$$




$$
\begin{aligned}
& =\sum_{j=0}^{k-1} \frac{h^{j}}{j !} F_{(j)}(x) \sum_{i=0}^{k-1}(-1)^{k-1-i}\left(\begin{array}{c}
k-1 \\
i
\end{array}\right)(2 i-k+1)^{j}+o\left(h^{k-1}\right) \\
& =(2 h)^{k-1} F_{(k-1)}(x)+o\left(h^{k-1}\right),
\end{aligned}
$$

and so for all $x \in E$,

$$
\lim _{h \rightarrow 0} \Delta_{k-1}(x, 2 h ; F) /(2 h)^{k-1}=F_{(k-1)}(x),
$$

which contradicts the fact that (3.5) can exist at most on a set of measure zero and thus the proof is complete.

Theorem 3.2 shows that in Theorem 3.1 the condition $\lambda>k-1$ is necessary. However, the following theorem shows that this condition can be relaxed if the existence of $f_{([\lambda]) \text {, a }}$ is assumed.

Theorem 3.4. Let $k \in \mathbb{N}, p \in \mathbb{N}, p \leq k-1$ and let $f: \mathbb{R} \rightarrow \mathbb{R}$ be measurable. Let

$$
\Delta_{k}(x, u)=O\left(u^{p}\right) \quad \text { as } u \rightarrow 0,
$$

for each point $x$ in a set $E$. If $f_{(p), a}$ exists finitely on $E$ then $f_{(p)}$ exists a.e. on $E$.

We need the following lemma.

Lemma 3.5. Let $k \in \mathbb{N}, p \in \mathbb{N}$ and let $f: \mathbb{R} \rightarrow \mathbb{R}$ be measurable. Let

$E=E_{m}=\left\{x: f_{(p), \mathrm{a}}(x)\right.$ exists finitely and

$$
\left.\left|\Delta_{k}(x, u)\right|<m|u|^{p} \text { for } 0<|u|<1 / m\right\} .
$$

Then $f_{(p)}$ exists a.e. on E.

Proof. Let $x_{0} \in E$ be a point of outer density of $E$. We suppose

$$
x_{0}=0=f\left(x_{0}\right)=f_{(1), \mathrm{a}}\left(x_{0}\right)=\ldots=f_{(p), \mathrm{a}}\left(x_{0}\right) .
$$

Let $0<\varepsilon<1$. Let

$$
G=\left\{x:|f(x)| \leq \varepsilon|x|^{p} / p !\right\} .
$$

Then $G$ is measurable and $0 \in G$ is a point of density of $G$. Set $H=E \cap G$. Then 0 is a point of outer density of $H$. Let $0<\eta<\varepsilon /(2 k)$. Then by Lemma 2.1 there is $\delta>0$ such that if $0<u<\delta$ then

$$
\mu^{*}(B)>(1-\eta) u, \quad \mu^{*}\left(C_{j}\right)>(1-\eta) u,
$$

where

$$
\begin{aligned}
B & =\{v \in[u, 2 u]:(u+v) / 2 \in H\}, \\
C_{j} & =\{v \in[u, 2 u]: v+j(u-v) / k \in H\}, \quad 0 \leq j \leq k-1 .
\end{aligned}
$$


Fix $u \in(0, \min (\delta, 1 / m))$. Let

$$
\begin{aligned}
& S=\left\{v \in[u, 2 u]:\left|\Delta_{k}((u+v) / 2,(u-v) / k)\right|<m|(u-v) / k|^{p}\right\}, \\
& T_{j}=\left\{v \in[u, 2 u]:|f(v+j(u-v) / k)| \leq \varepsilon|v+j(u-v) / k|^{p} / p !\right\}, \\
& 0 \leq j \leq k-1 .
\end{aligned}
$$

Since $f$ is measurable, $S$ and $T_{j}$ are measurable. Also $B \subset S, C_{j} \subset T_{j}$ and hence

$$
\mu(S)>(1-\eta) u, \quad \mu\left(T_{j}\right)>(1-\eta) u
$$

Therefore

$$
\mu\left(\bigcap_{j}\left(S \cap T_{j}\right)\right)>(1-2 k \eta) u>(1-\varepsilon) u .
$$

Hence

$$
\left(\bigcap_{j}\left(S \cap T_{j}\right)\right) \cap(u, u+\varepsilon u) \neq \emptyset .
$$

Choose $v \in\left(\bigcap_{j}\left(S \cap T_{j}\right)\right) \cap(u, u+\varepsilon u)$. Then $0<v-u<\varepsilon u<u<1 / m$ and so

$$
\left|\Delta_{k}((u+v) / 2,(u-v) / k)\right|<m|(u-v) / k|^{p}<m(\varepsilon u)^{p},
$$

which gives

$$
\left|\sum_{j=0}^{k}(-1)^{k-j}\left(\begin{array}{l}
k \\
j
\end{array}\right) f((u+v) / 2+(j-k / 2)(u-v) / k)\right|<m(\varepsilon u)^{p} .
$$

Hence

$$
|f(u)|<m(\varepsilon u)^{p}+\sum_{j=0}^{k-1}\left(\begin{array}{c}
k \\
j
\end{array}\right)|f(v+j(u-v) / k)| .
$$

Since $v \in T_{j}$ for $0 \leq j \leq k-1$,

$$
\begin{aligned}
|f(u)| & <m(\varepsilon u)^{p}+\sum_{j=0}^{k-1}\left(\begin{array}{c}
k \\
j
\end{array}\right) \varepsilon|v+j(u-v) / k|^{p} / p ! \\
& \leq m(\varepsilon u)^{p}+(\varepsilon / p !) \sum_{j=0}^{k-1}\left(\begin{array}{c}
k \\
j
\end{array}\right)(3 u)^{p} \\
& \leq \varepsilon\left[m+\left(3^{p} / p !\right) \sum_{j=0}^{k-1}\left(\begin{array}{l}
k \\
j
\end{array}\right)\right] u^{p} .
\end{aligned}
$$

This shows that $f(u) / u^{p} \rightarrow 0$ as $u \rightarrow 0+$.

It can be similarly shown that $f(u) / u^{p} \rightarrow 0$ as $u \rightarrow 0-$. This completes the proof of the lemma. 
Proof of Theorem 3.4. For each positive integer $m$, let

$E_{m}=\left\{x: f_{(p), \mathrm{a}}(x)\right.$ exists finitely and

$$
\left.\left|\Delta_{k}(x, u)\right|<m|u|^{p} \text { for } 0<|u|<1 / m\right\} .
$$

Then $\left\{E_{m}\right\}$ is a non-decreasing sequence and $E \subset \bigcup_{m} E_{m}$. By Lemma 3.5, $f_{(p)}$ exists a.e. on $E_{m}$ and so the result follows.

Corollary 3.6. Let $p \in \mathbb{N}$, let $f: \mathbb{R} \rightarrow \mathbb{R}$ be measurable and let $f(x)=0$ for $x \in E \subset \mathbb{R}$. If

$$
f(x+u)-f(x-u)=O\left(u^{p}\right)
$$

or

$$
f(x+u)+f(x-u)=O\left(u^{p}\right)
$$

for $x \in E$, then $f_{(p)}$ exists a.e. on $E$.

Proof. Let

$$
\begin{aligned}
& E_{1}=\left\{x \in E: f(x+u)-f(x-u)=O\left(u^{p}\right)\right\}, \\
& E_{2}=\left\{x \in E: f(x+u)+f(x-u)=O\left(u^{p}\right)\right\} .
\end{aligned}
$$

Then $E=E_{1} \cup E_{2}$. Let $D_{i}$ be the set of all points of $E_{i}$ which are also points of outer density of $E_{i}, i=1,2$. Then $f_{(p), \mathrm{a}}(x)=0$ for $x \in D_{1} \cup D_{2}$. Also

$$
\begin{array}{ll}
\Delta_{1}(x, u)=O\left(u^{p}\right) & \text { as } u \rightarrow 0 \text { for } x \in D_{1}, \\
\Delta_{2}(x, u)=O\left(u^{p}\right) & \text { as } u \rightarrow 0 \text { for } x \in D_{2} .
\end{array}
$$

Hence if $p=1$ then by Theorem 3.1, $f^{\prime}$ exists finitely a.e. on $D_{1}$ and by Theorem 3.4, $f^{\prime}$ exists finitely a.e. on $D_{2}$ and hence $f^{\prime}$ exists a.e. on $E$. If $p \geq 2$ then by Theorem 3.1, $f_{(p)}$ exists finitely a.e. on $D_{1}$ and on $D_{2}$ and hence $f_{(p)}$ exists finitely a.e. on $E$.

The above corollary is a generalization of Lemma 11 of [8, p. 268], since we are not assuming the measurability of $E$.

Theorem 3.4 can further be extended to

Theorem 3.7. Let $k \in \mathbb{N}, p \in \mathbb{N}, p \leq k-1$ and let $f: \mathbb{R} \rightarrow \mathbb{R}$ be measurable. Let

$$
\Delta_{k}(x, u)=O\left(u^{p}\right) \quad \text { as } u \rightarrow 0
$$

for each point $x$ in a set $E$. If $f_{(p-1), \mathrm{a}}$ exists and

$$
-\infty<\underline{f}_{(p), \mathrm{a}} \leq \bar{f}_{(p), \mathrm{a}}<\infty \quad \text { on } E
$$

then $f_{(p-1)}$ exists and

$$
-\infty<\underline{f}_{(p)} \leq \bar{f}_{(p)}<\infty \quad \text { a.e. on } E .
$$

P r o of. The first part follows from Theorem 3.4. The proof of the second part is similar to that of Theorem 3.4. We give a sketch. The corresponding 
sets in Lemma 3.5 are in this case given by

$$
\begin{aligned}
& E_{m}=\left\{x: f_{(p-1), \mathrm{a}}(x) \text { exists finitely, }\left|\Delta_{k}(x, u)\right|<m|u|^{p}\right. \\
& \left.\quad \text { for } 0<|u|<1 / m \text { and }-m<\underline{f}_{(p), \mathrm{a}}(x) \leq \bar{f}_{(p), \mathrm{a}}(x)<m\right\}
\end{aligned}
$$

with the assumption that

$$
\begin{aligned}
x_{0} & =0=f\left(x_{0}\right)=f_{(1), \mathrm{a}}\left(x_{0}\right)=\ldots=f_{(p-1), \mathrm{a}}\left(x_{0}\right), \\
G_{m} & =\left\{x:|f(x)| \leq m|x|^{p} / p !\right\}, \\
T_{j} & =\left\{v \in[u, 2 u]:|f(v+j(u-v) / k)| \leq m|v+j(u-v) / k|^{p} / p !\right\}, \\
& 0 \leq j \leq k-1,
\end{aligned}
$$

and the final step is

$$
|f(u)| \leq\left[\varepsilon m+m\left(3^{p} / p !\right) \sum_{j=0}^{k-1}\left(\begin{array}{l}
k \\
j
\end{array}\right)\right] u^{p}
$$

showing that $|f(u)|=O\left(u^{p}\right)$ as $u \rightarrow 0+$ and similarly $|f(u)|=O\left(|u|^{p}\right)$ as $u \rightarrow 0-$.

Corollary 3.8. Under the hypothesis of Theorem 3.7, if $f_{(p-1), a}$ exists and

$$
-\infty<\underline{f}_{(p), \mathrm{a}}(x) \leq \bar{f}_{(p), \mathrm{a}}(x)<\infty \quad \text { on } E
$$

then $f_{(p)}$ exists a.e. on $E$.

The proof follows from Theorems 3.7 and 2.2 .

\section{References}

[1] P. S. Bullen and S. N. Mukhopadhyay, Peano derivatives and general integrals, Pacific J. Math. 47 (1973), 43-58.

[2] A. Denjoy, Sur l'intégration des coefficients différentielles d'ordre supérieur, Fund. Math. 25 (1935), 273-326.

[3] H. Fejzic and C. E. Weil, Repairing the proof of a classical differentiation result, Real Anal. Exchange 19 (1993-94), 639-643.

[4] J. Marcinkiewicz, Sur les séries de Fourier, Fund. Math. 27 (1937), 38-69.

[5] J. Marcinkiewicz and A. Zygmund, On the differentiability of functions and summability of trigonometric series, ibid. 26 (1936), 1-43.

[6] S. N. Mukhopadhyay and S. Mitra, Measurability of Peano derivates and approximate Peano derivates, Real Anal. Exchange 20 (1994-95), 768-775.

[7] S. Saks, Theory of the Integral, Dover, 1964.

[8] E. M. Stein and A. Zygmund, On the differentiability of functions, Studia Math. 23 (1964), 247-283. 
[9] A. Zygmund, Trigonometric Series I, II, Cambridge Univ. Press, 1968.

Department of Mathematics

The University of Burdwan

Burdwan, 713104, India

Received 16 February 1995 